\title{
Thiol passivation of MWIR type II superlattice photodetectors
}

O. Salihoglu

A. Muti

A. Aydinli 


\title{
Thiol Passivation of MWIR Type II Superlattice Photodetectors
}

\author{
O. Salihoglu, A. Muti and A. Aydinli \\ Department of Physics, Bilkent University, 06800 Ankara, Turkey
}

\begin{abstract}
Poor passivation on photodetectors can result in catastrophic failure of the device. Abrupt termination of mesa side walls during pixel definition generates dangling bonds that lead to inversion layers and surface traps leading to surface leakage currents that short circuit diode action. Good passivation, therefore, is critical in the fabrication of high performance devices. Silicondioxide has been the main stay of passivation for commercial photodetectors, deposited at high temperatures and high RF powers using plasma deposition techniques. In photodetectors based on III-V compounds, sulphur passivation has been shown to replace oxygen and saturate the dangling bonds. Despite its effectiveness, it degrades over time. More effort is required to create passivation layers which eliminate surface leakage current. In this work, we propose the use of sulphur based octadecanethiol (ODT), $\mathrm{CH}_{3}\left(\mathrm{CH}_{2}\right)_{17} \mathrm{SH}$, as a passivation layer for the $\mathrm{InAs} / \mathrm{GaSb}$ superlattice photodetectors that acts as a self assembled monolayer (SAM). ODT SAMs consist of a chain of 18 carbon atoms with a sulphur atom at its head. ODT Thiol coating is a simple process that consist of dipping the sample into the solution for a prescribed time. Excellent electrical performance of diodes tested confirm the effectiveness of the sulphur head stabilized by the intermolecular interaction due to van der Walls forces between the long chains of ODT SAM which results in highly stable ultrathin hydrocarbon layers without long term degradation.
\end{abstract}

Keywords: Superlattice, Photodetector, InAs/GaSb, ODT, SAM, Thiol, Sulfide, Passivation

\section{INTRODUCTION}

Passivation is a key process for type-II superlattice (T2SL) InAs/GaSb photodetectors for competitive performance. Passivation heals the surface of the freshly etch mesa side walls to suppress surface leakage currents and protects it against environmental effects. Surface leakage currents mostly originate from surface states due to dangling bonds, inversion layers and interfacial traps which are generated due to the abrupt termination of the crystal structure. Various passivation methods such as sulfide passivation [1], dielectric layer deposition [2, 3], overgrowth with wide bandgap materials [4], polymer film coating [5] have been proposed to overcome these problems. Sulphur passivation is an effective passivation method and is easy to apply but the effect of passivation is temporary making it impractical [6]. Sulphur replaces oxygen in conductive surface oxides $\left(\mathrm{Ga}_{2} \mathrm{O}_{3}, \mathrm{In}_{2} \mathrm{O}_{3}, \mathrm{GaO}_{2}\right.$... etc) and saturates the dangling bonds [7]. Sulphur is generally applied using ammonium sulfide, sodium sulfide or zinc sulfide solutions. Alternatively, electrochemical sulphur passivation (ECP) treatment has been tried [1]. Different types of capping layers has been used to increase long term stability [8]. Besides long term stability issues, sulphur solutions can etch GaSb layers with rates as high as 120 $\mathrm{nm} /$ minute [9] resulting in damaged $\mathrm{p}^{+} \mathrm{GaSb}$ layer which creates very high series resistance and reduces device optical performance. In this study, we propose to use octadecanethiol (ODT), $\mathrm{CH}_{3}\left(\mathrm{CH}_{2}\right)_{17} \mathrm{SH}$, a sulphur containing self assembled monolayer (SAM), as a passivation layer for the InAs/GaSb superlattice photodetectors. ODT SAMs contains 18 carbon long chain and a reactive sulphur head (S-H). Active sulphur end of the thiol replaces oxygen atoms and creates sulphur bonds on the surface. It cleans the surface from conductive native oxides and satisfies dangling bonds[10-12]. Although thiol is mostly studied on GaAs [13-16] surfaces, some studies on InP [17], GaP [18], and InAs [11, 12] and other III-V compounds have also been reported. McGuiness et al. has shown that long chained octadecanethiol strongly reduces As and Ga oxides and prevents subsequent surface oxide re-growth [16] using X-ray photoelectron spectroscopy (XPS). Knoben et al., showed that ODT can form a well ordered monolayer on InAs and reduce surface oxidation [11, 12]. Work on

Infrared Technology and Applications XXXIX, edited by Bjørn F. Andresen, Gabor F. Fulop,

Charles M. Hanson, Paul R. Norton, Proc. of SPIE Vol. 8704, 87040T - (c) 2013 SPIE

CCC code: $0277-786 \mathrm{X} / 13 / \$ 18 \cdot$ doi: $10.1117 / 12.2015521$

Proc. of SPIE Vol. 8704 87040T-1 
InP photodiodes has demonstrated suppression of dark currents due to ODT SAM [17]. Unlike other sulphur passivations, SAMs thiol does not damage III-V surfaces [11] and long chain length of the ODT SAM increases thermodynamic stability [19] where intermolecular forces lead to formation of a skin-like hydrocarbon layer. SAM thiol works as well as sulphur passivation without problems of the sulphur passivation.

\section{EXPERIMENTAL}

The SL structure was designed for MWIR operation with design cutoff wavelength of $5 \mu \mathrm{m}$. Figure 1a. shows structure of the $\mathrm{p}$-i-n design superlattice crystal with corresponding thicknesses and doping concentrations. The pin part includes 140 periods 8 monolayers (MLs) of InAs/8 MLs of GaSb:Be $\left(\mathrm{p}=1.5 \times 10^{17}\right.$ $\mathrm{cm}^{-3}$ ) p-type layers, 40 periods 8 MLs of InAs / 8 MLs of GaSb i-layers, 60 periods 8 MLs of InAs:Te (n: 5x10 17 $\left.\mathrm{cm}^{-3}\right) / 8$ MLs of GaSb n-type layers. $1000 \mathrm{~nm} \mathrm{GaSb}: \mathrm{Be}\left(\mathrm{p}=1.0 \times 10^{17} \mathrm{~cm}^{-3}\right)$ and $20 \mathrm{~nm}$ InAs:Te $\left(\mathrm{n}: 5 \times 10^{17} \mathrm{~cm}^{-3}\right)$ layers introduced as $\mathrm{p}$ contact and $\mathrm{n}$ contact layers, respectively. Fotodetectors with mesa sizes from $100 \mathrm{x} 100$ $\mu \mathrm{m}^{2}$ to $700 \times 700 \mu \mathrm{m}^{2}$ are defined using phosphoric acid based solution. Ohmic contacts were deposited by evaporating $5 \mathrm{~nm}$ titanium and $200 \mathrm{~nm}$ gold [20]. For ODT passivation, samples were immersed in a solution of $\mathrm{HCl}$ in isopropanol (3M) for $1 \mathrm{~min}$. and rinsed in isopropanol for $5 \mathrm{~min}$. prior to immersion in $1 \mathrm{mM}$ solution of ODT (Aldrich, 99\%) in ethanol and was left there for 48 hours at $60{ }^{\circ} \mathrm{C}$. Then the sample was rinsed with ethanol and isopropanol and dried with dry $\mathrm{N}_{2}$. Fig. $1 \mathrm{~b}$ shows schematic of ODT on the superlattice surface. ODT molecules form smooth continuous crystalline like packed hydrocarbon layer with tilt angle of $\sim 30^{\circ}$ from the surface normal [12]. For the electrical and optical measurements, samples were bonded to a 40 pin DIP chip carrier (figure 1c).

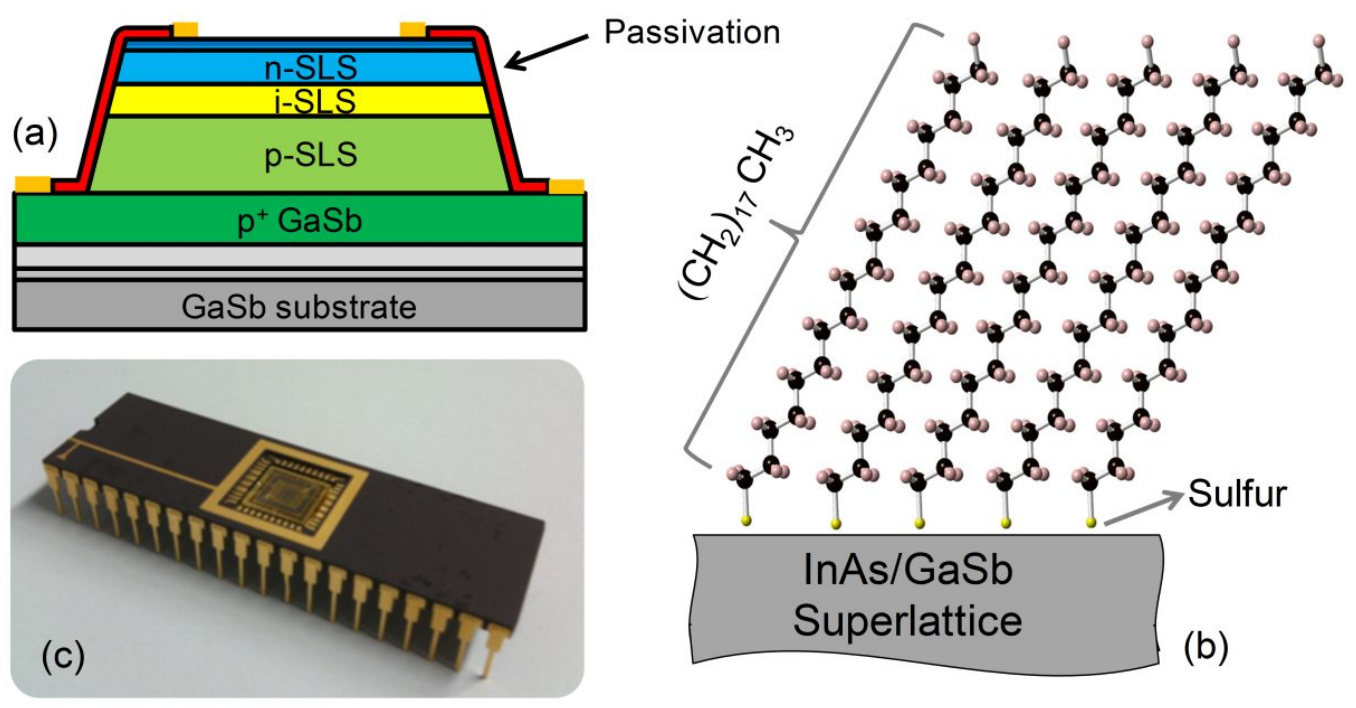

Figure1. (a) Structure of the single pixel photodetector. (b) Schematic of the ODT on the superlattice surface. (c) Image of the sample bonded to a 40 pin DIP chip carrier.

\section{RESULTS AND DISCUSSIONS}

Dark current and dynamic resistance measurements have been done at $77 \mathrm{~K}$ to investigate electrical performance of the ODT SAM. We have used unpassivated and $\mathrm{SiO}_{2}$ passivated control samples along with ODT passivated samples. The photodetectors were put into an aluminum box and immersed into liquid nitrogen filled container. HP4142OA source-measure unit has been used to measure dark current voltage-current characteristics of the samples. Figure $2 \mathrm{a}$ shows the measured dark current density vs. applied bias voltage 
characteristics of the photodiodes at $77 \mathrm{~K}$. Passivated detectors show at least two orders of magnitude reduction in dark current density compared with unpassivated detectors. Dark current density (at $-0.1 \mathrm{~V}$ ), reduced from 1.3 x $10^{-4} \mathrm{~A} / \mathrm{cm}^{2}$ to $2.2 \times 10^{-7} \mathrm{~A} / \mathrm{cm}^{2}$ and $3.9 \times 10^{-8} \mathrm{~A} / \mathrm{cm}^{2}$ for $\mathrm{SiO}_{2}$ passivated and ODT passivated diodes respectively. These measurements yield $\mathrm{R}_{0} \mathrm{~A}$ product values of $1.1 \times 10^{3} \Omega \cdot \mathrm{cm}^{2}, 5.2 \times 10^{5} \Omega \cdot \mathrm{cm}^{2}$ and $3.9 \times 10^{6}$ $\Omega \cdot \mathrm{cm}^{2}$ for the unpassivated, $\mathrm{SiO}_{2}$ and ODT SAM passivated photodetectors, respectively (Fig. 2b).

Photodetectors with ODT passivation show at least an order of magnitude lower dark current than $\mathrm{SiO}_{2}$ passivated photodetectors up to $0.3 \mathrm{~V}$ reverse bias. Beyond $-0.3 \mathrm{~V}$, slope of the dark current in ODT passivated detectors increase. At low temperatures, currents in the photodetector are diffusion limited near zero bias. At larger biases, our results suggest that trap assisted currents are dominant for ODT passivation. Thickness of the ODT passivation is only $2 \mathrm{~nm}$, which suggest that for higher biases the traps at the interface of the passivation layer may still effect the performance. For zero or small reverse bias, application of ODT SAM is a very effective way for passivation of the type-II InAs/GaSb p-i-n photodetectors. The prominent reduction in dark current due to ODT SAM passivation is very encouraging when compared with recently published type-II $\mathrm{InAs} / \mathrm{GaSb}$ passivation works [6, 21].
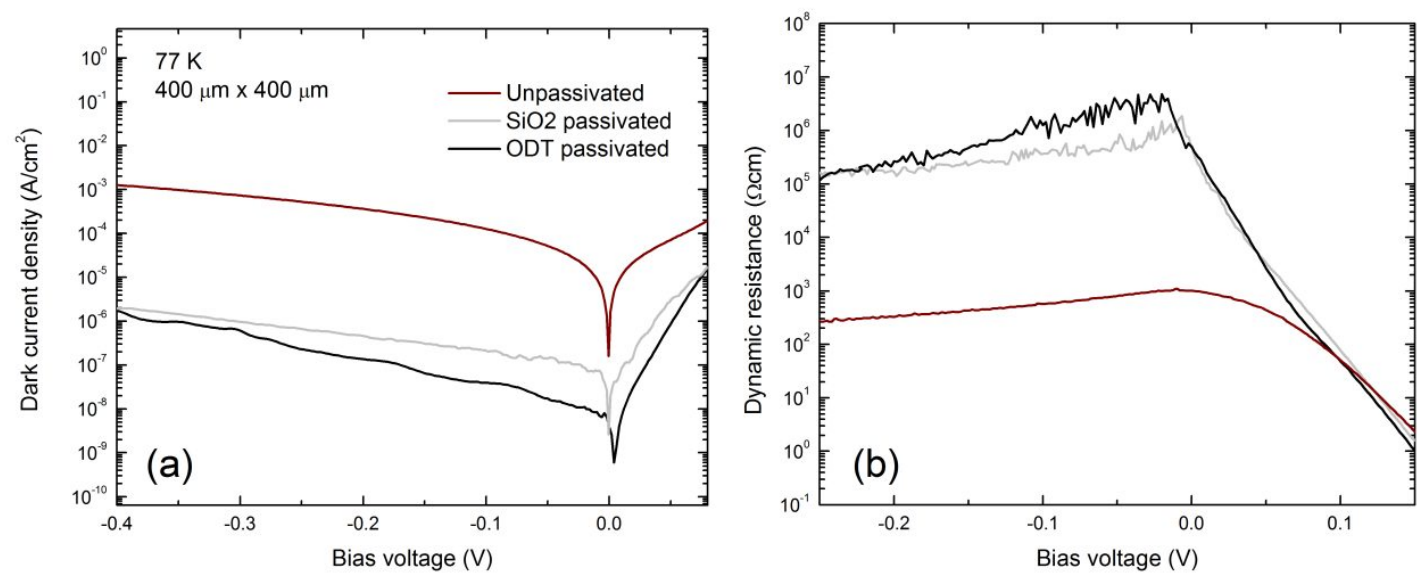

Figure 2. (a) Dark current density vs. applied bias of unpassivated $\mathrm{SiO}_{2}$ and ODT passivated $400 \mathrm{x} 400 \mu \mathrm{m}$ diodes at $77 \mathrm{~K}$. (b) Zero bias differential resistance vs. applied bias voltage characteristics for the unpassivated, $\mathrm{SiO}_{2}$ and ODT passivated samples at $77 \mathrm{~K}$.

Dark current densities versus inverse temperature under -0.1V bias are shown in Figure 3. Diffusion current is dominant for the temperatures higher than $100 \mathrm{~K}$ and generation-recombination current is dominant for temperatures lower than $100 \mathrm{~K}$. For the temperatures lower than $100 \mathrm{~K}$, ODT SAM passivated diodes show lower dark currents than unpassivated diodes. This suggests that the native oxides are cleaned, surface states are reduced and surface channel currents are lowered by the ODT SAM passivation. Arhenious type behavior for temperatures higher than $100 \mathrm{~K}$, yielded activation energy of $0.23 \mathrm{eV}$ which is close to the band gap of the superlattice material. For the lower temperatures, generation recombination (G-R) current becomes dominant. Passivated photodiodes show reduced G-R currents, which may originate both from the bulk and the surface. Figure 3 also shows the summary of different stages of aging. Measurements indicate that ODT SAM does not show any sign of aging even after 65 days. Inset of figure 3 shows dark current density vs. days at $77 \mathrm{~K}$ under inverse $0.1 \mathrm{~V}$ bias. Small fluctuations are due to the noise floor of our instrument. Strong intermolecular interactions between long chains of octadecanethiol molecules form a closely packed and thermodynamically stable skin-like layer.

Surface chemistry of the freshly etched surface has large impact on the performance of the superlattice photodetector. Chemically reactive In, As, $\mathrm{Ga}$ and $\mathrm{Sb}$ atoms are easily oxidized by atmospheric effects after the mesa definition process (etching). This causes formation of very thin conductive native oxide layers at the surface which are labeled conductive channels and lead to leakage. Sulphur is chemically more reactive than 
oxygen so that replaces oxygen to form electrically stable surface sulfides. These reactions may be called interfacial self-cleaning reactions which are confirmed by XPS measurements $[11,16]$. The sulphur interaction with the InAs creates $\operatorname{In}_{x} S_{y}$ and $\mathrm{As}_{\mathrm{x}} \mathrm{S}_{\mathrm{y}}\left(\mathrm{InAs}+2 \mathrm{HS}=\operatorname{In}_{x^{\prime}} \mathrm{S}_{\mathrm{y}^{\prime}}+\mathrm{As}_{\mathrm{x}^{\prime \prime}} \mathrm{S}_{\mathrm{y}^{\prime \prime}}+\mathrm{H}_{2}\right)$ and interaction with the GaSb creates $\mathrm{Ga}_{\mathrm{x}} \mathrm{S}_{\mathrm{y}}$ and $\mathrm{Sb}_{\mathrm{x}} \mathrm{S}_{\mathrm{y}}\left(\mathrm{GaSb}+2 \mathrm{HS}=\mathrm{Ga}_{\mathrm{x}} \mathrm{S}_{\mathrm{y}^{\prime}}+\mathrm{Sb}_{\mathrm{x}^{\prime \prime}} \mathrm{S}_{\mathrm{y}^{\prime \prime}}+\mathrm{H}_{2}\right)$ on the surface. Finally, strong intermolecular interactions between long chains of ODT molecules form a closely packed and thermodynamically stable skin-like ultrathin protective layer. These results suggest that this process is beneficial for focal plane array (FPA) and LWIR applications.

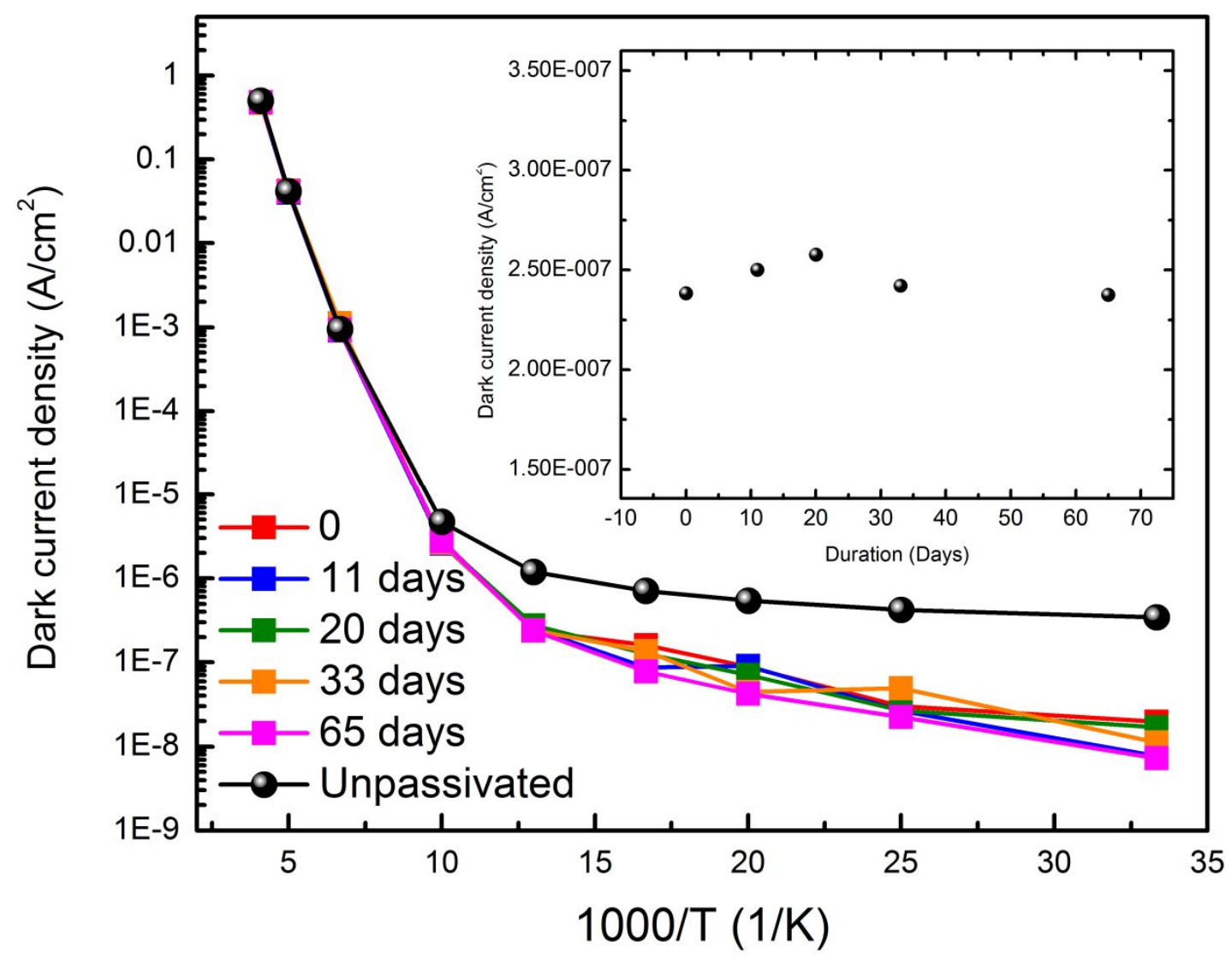

Figure 3. Temperature dependent dark current density for unpassivated and ODT SAM passivated type-II InAs/GaSb superlattice $400 \times 400 \mu \mathrm{m}$ photodiodes at inverse $0.1 \mathrm{~V}$ bias voltage. Inset shows dark current density vs. days at $77 \mathrm{~K}$ under inverse $0.1 \mathrm{~V}$ bias. Small fluctuations are due to the noise floor of our instrument.

\section{CONCLUSIONS}

We have demonstrated an alternative passivation method for the type-II InAs/GaSb photodetectors with cutoff wavelength at $5.1 \mu \mathrm{m}$ (MWIR). Our result showed that ODT SAM self cleans the surface from native oxides and satisfies surface states by replacing oxygen atoms with active sulphur. Passivated detectors show at least two orders of magnitude reduction in dark current density compared with unpassivated detectors. Dark current density (at $-0.1 \mathrm{~V}$ ), reduced from $1.3 \times 10^{-4} \mathrm{~A} / \mathrm{cm}^{2}$ to $2.2 \times 10^{-7} \mathrm{~A} / \mathrm{cm}^{2}$ and $3.9 \times 10^{-8} \mathrm{~A} / \mathrm{cm}^{2}$ for $\mathrm{SiO}_{2}$ passivated and ODT passivated diodes respectively. These measurements yield $\mathrm{R}_{0} \mathrm{~A}$ product values of $1.1 \times 10^{3}$ $\Omega \cdot \mathrm{cm}^{2}, 5.2 \times 10^{5} \Omega \cdot \mathrm{cm}^{2}$ and $3.9 \times 10^{6} \Omega \cdot \mathrm{cm}^{2}$ for the unpassivated, $\mathrm{SiO}_{2}$ and ODT SAM passivated photodetectors, respectively. Temperature dependent dark current and dynamic resistance-area product as a 
perimeter to area ratio measurements revealed that passivation prevents photodetectors from being dominated by surface currents for temperatures lower than 100K. Devices show Arrhenius type of behavior at higher temperatures, an indication that dominant current is bulk diffusion current for high temperatures. The dark current performance of photodiodes did not degrade for 65 days after the passivation, indicating a long-term stability. This work shows that ODT SAM is a very effective passivation method for near zero bias of T2SL photodiodes.

\section{REFERENCES}

[1] E. Plis, J. B. Rodriguez, S. J. Lee, and S. Krishna, "Electrochemical sulphur passivation of InAs/GaSb strain layer superlattice detectors," Electronics Letters, vol. 42, pp. 1248-1249, Oct 122006.

[2] A. Gin, Y. J. Wei, J. J. Bae, A. Hood, J. Nah, and M. Razeghi, "Passivation of type IIInAs/GaSb superlattice photodiodes," Thin Solid Films, vol. 447, pp. 489-492, Jan 302004.

[3] O. Salihoglu, A. Muti, K. Kutluer, T. Tansel, R. Turan, and A. Aydinli, "Atomic Layer Deposited $\mathrm{Al}_{2} \mathrm{O}_{3}$ Passivation of Type-II InAs/GaSb Superlattice Photodetectors " J. Appl. Phys., vol. 111, p. 074509, 2012.

[4] R. Rehm, M. Walther, F. Fuchs, J. Schmitz, and J. Fleissner, "Passivation of InAsA/(GaIn)Sb shortperiod superiattice photodiodes with $10 \mathrm{mu}$ m cutoff wavelength by epitaxial overgrowth with AlxGa1xAsySb1-y," Applied Physics Letters, vol. 86, p. 173501, Apr 252005.

[5] A. Hood, P. Y. Delaunay, D. Hoffman, B. M. Nguyen, Y. J. Wei, M. Razeghi, and V. Nathan, "Near bulk-limited $\mathrm{R}(0) \mathrm{A}$ of long-wavelength infrared type-II InAs/GaSb superlattice photodiodes with polyimide surface passivation," Applied Physics Letters, vol. 90, p. 233513, Jun 42007.

[6] E. Plis, M. N. Kutty, S. Myers, H. S. Kim, N. Gautam, L. R. Dawson, and S. Krishna, "Passivation of long-wave infrared InAs/GaSb strained layer superlattice detectors," Infrared Physics \& Technology, vol. 54, pp. 252-257, May 2011.

[7] J. Hoffmann, T. Lehnert, D. Hoffmann, and H. Fouckhardt, "Advantages and disadvantages of sulphur passivation of InAs/GaSb superlattice waveguide photodiodes," Semiconductor Science and Technology, vol. 24, p. 065008, Jun 2009.

[8] J. V. Li, S. L. Chuang, O. V. Sulima, and J. A. Cox, "Passivation of AlGaAsSb/InGaAsSb/GaSb photodiodes using aqueous (NH4)(2)S solution and polyimide encapsulation," Journal of Applied Physics, vol. 97, p. 104506, May 152005.

[9] A. Hood, Y. wei, A. Gin, M. Razeghi, M. Z. Tidrow, and V. Nathan, "Passivation of Type-II InAs/GaSb Superlattice Photodetectors," Proc. of SPIE, vol. 5732, pp. 316-325, 2005.

[10] C. J. Spindt, D. Liu, K. Miyano, P. L. Meissner, T. T. Chiang, T. Kendelewicz, I. Lindau, and W. E. Spicer, "Vacuum Ultraviolet Photoelectron-Spectroscopy of (Nh4)2s-Treated Gaas (100) Surfaces," Applied Physics Letters, vol. 55, pp. 861-863, Aug 281989.

[11] D. Y. Petrovykh, J. C. Smith, T. D. Clark, R. Stine, L. A. Baker, and L. J. Whitman, "Self-Assembled Monolayers of Alkanethiols on InAs," Langmuir, vol. 25, pp. 12185-12194, Oct 202009.

[12] W. Knoben, S. H. Brongersma, and M. Crego-Calama, "Preparation and Characterization of Octadecanethiol Self-Assembled Monolayers on Indium Arsenide (100)," Journal of Physical Chemistry C, vol. 113, pp. 18331-18340, Oct 222009.

[13] S. Ye, G. F. Li, H. Noda, K. Uosaki, and M. Osawa, "Characterization of self-assembled monolayers of alkanethiol on GaAs surface by contact angle and angle-resolved XPS measurements," Surface Science, vol. 529, pp. 163-170, Apr 12003.

[14] Y. Jun, X. Y. Zhu, and J. W. P. Hsu, "Formation of alkanethiol and alkanedithiol monolayers on GaAs(001)," Langmuir, vol. 22, pp. 3627-3632, Apr 112006.

[15] H. A. Budz and R. R. LaPierre, "Properties of octadecanethiol self-assembled monolayers deposited on GaAs from liquid and vapor phases," Journal of Vacuum Science \& Technology A, vol. 26, pp. 14251431, Nov 2008.

[16] C. L. McGuiness, A. Shaporenko, M. Zharnikov, A. V. Walker, and D. L. Allara, "Molecular selfassembly at bare semiconductor surfaces: Investigation of the chemical and electronic properties of the alkanethiolate-GaAs(001) interface," Journal of Physical Chemistry C, vol. 111, pp. 4226-4234, Mar 22 2007. 
[17] M. Schvartzman, V. Sidorov, D. Ritter, and Y. Paz, "Passivation of InP surfaces of electronic devices by organothiolated self-assembled monolayers," Journal of Vacuum Science \& Technology B, vol. 21, pp. 148-155, Jan-Feb 2003.

[18] R. Flores-Perez, D. Y. Zemlyanov, and A. Ivanisevic, "Lithography on GaP(100) surfaces," Surface Science, vol. 602, pp. 1993-1998, Jun 12008.

[19] J. C. Love, L. A. Estroff, J. K. Kriebel, R. G. Nuzzo, and G. M. Whitesides, "Self-assembled monolayers of thiolates on metals as a form of nanotechnology," Chemical Reviews, vol. 105, pp. 11031169, Apr 2005.

[20] O. Salihoglu, A. Muti, K. Kutluer, T. Tansel, R. Turan, and A. Aydinli, "Skin-Like Self-Assembled Monolayers on InAs/GaSb Superlattice Photodetectors," J. Phys. D: Appl. Phys., vol. 45, p. 365102, 2012.

[21] H. S. Kim, E. Plis, A. Khoshakhlagh, S. Myers, N. Gautam, Y. D. Sharma, L. R. Dawson, S. Krishna, S. J. Lee, and S. K. Noh, "Performance improvement of InAs/GaSb strained layer superlattice detectors by reducing surface leakage currents with SU-8 passivation," Applied Physics Letters, vol. 96, p. 033502, Jan 182010. 